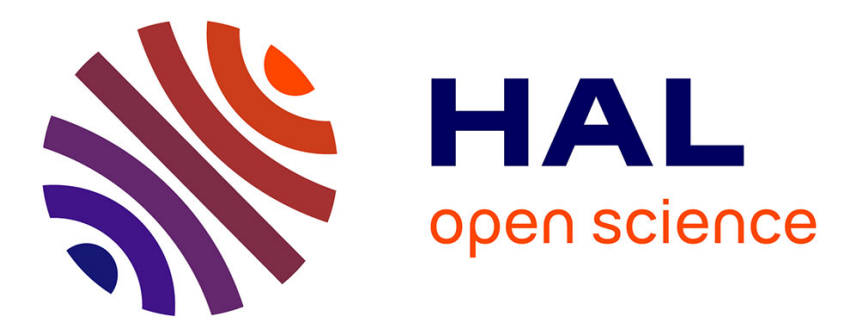

\title{
Le délit de prise illégale d'intérêts : de la sanction d'un devoir civique à la prévention d'un conflit d'intérêts
}

\author{
Yvonne Muller-Lagarde
}

\section{To cite this version:}

Yvonne Muller-Lagarde. Le délit de prise illégale d'intérêts: de la sanction d'un devoir civique à la prévention d'un conflit d'intérêts. Archives de politique criminelle, 2017, Nouveaux enjeux de la délinquance économique et financière, 39, pp.41-54. 10.3917/apc.039.0041 hal-01814562

\section{HAL Id: hal-01814562 \\ https://hal.parisnanterre.fr/hal-01814562}

Submitted on 15 Nov 2019

HAL is a multi-disciplinary open access archive for the deposit and dissemination of scientific research documents, whether they are published or not. The documents may come from teaching and research institutions in France or abroad, or from public or private research centers.
L'archive ouverte pluridisciplinaire $\mathbf{H A L}$, est destinée au dépôt et à la diffusion de documents scientifiques de niveau recherche, publiés ou non, émanant des établissements d'enseignement et de recherche français ou étrangers, des laboratoires publics ou privés. 


\title{
LE DELIT DE PRISE ILLEGALE D'INTERETS : DE LA SANCTION D'UN DEVOIR CIVIQUE A LA PREVENTION D'UN CONFLIT D'INTERETS
}

\author{
par \\ YVONNE MUlLER-LAGARDE \\ Co-Directrice du Centre de droit pénal et de criminologie (CDPC), \\ Université Paris Nanterre
}

Le délit de prise illégale d'intérêts, garant de la probité de l'agent public, porte en son sein le mythe de l'intérêt général, fondateur de l'Etat-Nation et d'une administration dévouée. Un siècle après sa création, sous le titre de «délit d'ingérence », par le code pénal napoléonien (1810), et plus de vingt ans après sa réécriture, sous son titre actuel, dans le code pénal (1992), le délit se pose encore comme le garant d'une fonction publique vertueuse, seule à même de maintenir "l'adhésion de nos concitoyens aux fondements de la République » et partant, de contribuer à la "solidité de notre démocratie ${ }^{1}$.

Toutefois, étroitement associé à la construction de l'Etat-moderne et à l'émergence d'une véritable fonction publique, le délit de prise illégale d'intérêts porte inévitablement les traces de leurs mutations marquées par la réduction de la «chose publique». En témoigne la hiérarchie des intérêts protégés par les différents codes pénaux. Alors que les codes de 1791 et de 1810 sont centrés sur la «chose publique » et la défense de l'Etat, le nouveau code pénal de 1992, inspiré par les droits de l'homme, renverse la hiérarchie des intérêts protégés en plaçant au sommet la protection des personnes; "Les crimes et délits contre la Nation, l'Etat et la paix publique " viennent désormais en troisième position, après les atteintes aux personnes et aux biens ${ }^{2}$. C'est désormais « $\grave{a}$ travers l'individu, ses intérêts et ses valeurs qu'est construit l'essentiel du bien commun» révélant, ce qu'un auteur appelle une "dépolitisation de l'ordre public $»^{3}$. Au-delà de la forme juridique, ce renversement est l'expression d'une transformation de l'Etat moderne et de ses formes d'action. La souveraineté étatique, remise en cause, doit être repensée. Confronté à un phénomène de " décentralité » accentué par la construction de l'Europe et, plus globalement par la mondialisation, l'Etat tend à perdre la maîtrise de son environnement comme en témoigne le flou des frontières juridiques et la pluralité des modes de

\footnotetext{
${ }^{1}$ A-M. EsCOFFIER, Sénat, Rapport $n^{\circ} 519$ (2009-2010) fait au nom de la Commission des lois et déposé le 2 juin 2010, Partie III.

${ }^{2}$ P. LASCOUMES et A. DEPAIGNE, «Catégoriser l'ordre public : la réforme du code pénal français de 1992 », Genèses, 1997, vol. 27, n 1, pp. 5 à 29, spéc. p. 16.

${ }^{3}$ Ibid., spéc. p. 23.
} 
gouvernance. Il appelle à un autre ordre juridique. A l'Etat moderne se substituent progressivement, depuis le début du $X X^{\text {ème }}$ siècle, d'autres figures de l'Etat: libéral, propulsif, providence, post-moderne etc.; S'imposerait aujourd'hui celle d'un Etat réflexif conçu comme un droit procédural et organisationnel qui, par ses modes d'actions, dépasse la fracture désormais factice entre le secteur public et le secteur privé ${ }^{4}$. C'est reconnaître que l'intérêt général peut être le fait tant d'acteurs publics que d'acteurs privés.

Cette mutation de l'Etat impose une réflexion nouvelle sur le délit de prise illégale d'intérêts. Ce dernier puise ses racines dans la naissance, au XIX ${ }^{\text {ème }}$ siècle d'une fonction publique associée à l'Etat moderne et l'émergence d'une distinction entre l'intérêt public porté par l'Etat et les intérêts privés des particuliers; parce qu'il prend sens dans le cœur même de cette distinction, le délit de prise illégale d'intérêts en porte les évolutions mais se referme aussi sur ses contradictions. Le délit a évolué, dans sa définition, modifiée par le code pénal de 1992, mais aussi son interprétation jurisprudentielle révélant une sorte de « plasticité » ${ }^{5}$. En résulte une application jurisprudentielle non contrôlée qui a conduit les auteurs à qualifier l'infraction, peu précise et largement interprétée, de "délit de caractère stalinien ${ }^{6}$, "d'épée de Damoclès ${ }^{7}$, de " guillotine juridique ${ }^{8}$ ou encore de "délit poison ${ }^{9}$, de "bête noire des décideurs publics ${ }^{10}$ dont l'interprétation judiciaire excessive irait jusqu'à menacer certaines formes de vie politique $^{11}$ invitant le législateur à prendre sa plume ${ }^{12}$. Et l'on ne saurait se satisfaire, pour éviter la réflexion, de la constatation d'une répression certes réelle mais mesurée ${ }^{13}$, c'est-à-dire faible. Car à la critique d'un délit imprévisible s'ajoute alors celle de son inefficacité dans une période où l'exigence de vertu de l'agent public a rarement été aussi forte.

Aussi, la présente étude propose de montrer que le délit d'ingérence destiné, à l'origine, à sanctionner la violation par l'agent public de son devoir civique de probité (I) et rebaptisé en délit de prise illégale d'intérêts doit viser aujourd'hui à la prévention des conflits d'intérêts (II).

\footnotetext{
${ }^{4}$ L. ORTIZ, Nouvelles normativités et politiques publiques, Master IEP Toulousc, www. masternormativités.doc

${ }^{5}$ M. SEgOndS, « Prisc illćgale d'intérêts », Rép. Dalloz, 2017, p. 3, §7.

${ }^{6}$ G. Carcassonne, AN, Groupe de travail sur la prévention des conflits d'intérêts, audition du 9 déc. 2010.

${ }^{7}$ B. FLeURY, «Prise illégale d'intérêts: Ic grand retour de l'intention? », JCP A, n 45-46, nov. 2013, note s/Poitiers, 16 mai 2013, n 13/00170.

${ }^{8}$ W. Jeandidier, "La prise illégale d'intérêts indirecte par un élu ", note s/Cass.crim. 27 nov. 2002, $\mathrm{n}^{\circ} 7066, J C P . \mathrm{A}, \mathrm{n}^{\circ} 25,16$ juin 2003,1589, p. 821.

${ }^{9} \mathrm{~W}$. Jeandidier, art. préc.

${ }^{10}$ Nathalie LaVAL, "La prise illégale d'intérêts ", Droit Adm. n 6, Juin 2003, chron. 10

$"$ V. B. FLEURY, « Nul ne peut servir deux maitres à la fois (...) ", JCP A. 2010.act.539.

${ }^{12}$ Y. Mayaud, Cahiers du Cons. Const., avril 2013, n 39, Chron. 12, note s/ Cass., crim., 30 nov. $2011, n^{\circ} 11-90.093$.

${ }^{13}$ B. FLEURY, « Nul ne peut servir deux maitres à la fois (...) », art. préc.
} 


\section{LE DÉLIT D'INGÉRENCE OU LA SANCTION D'UN DEVOIR CIVIQUE}

C'est en réfléchissant à l'intelligence de la situation du fonctionnaire par rapport à l'Etat qui lui confie l'exercice de sa mission ${ }^{14}$ que Maurice Hauriou souligne, au début du $\mathrm{XX}^{\text {ème }}$ siècle qu'il y a autre chose dans la fonction publique qu'un pouvoir à exercer, il y a un "office social à remplir $»^{15}$. La thèse, alors validée par la jurisprudence, fait du fonctionnaire, soumis à une réglementation particulière, un «citoyen spécial $»^{16}$ chargé des fonctions publiques dans le seul intérêt général. La condition du fonctionnaire, ainsi magnifiée, est clairement dissociée du salarié du secteur privé. Elle trouve son fondement dans l'émergence progressive, avec la construction de l'Etat moderne, de la fonction publique et partant, du concept de fonctionnaire.

\section{A. L'émergence de la figure du fonctionnaire}

De fait, si la tradition administrative remonte à l'apparition de l'Etat-nation au $\mathrm{XVI}^{\text {ème }}$ siècle ${ }^{17}$, la fonction publique, institutionnalisée et idéalisée, émerge progressivement après la Révolution pour s'imposer au début du XIX ${ }^{\text {ème }}$ siècle. Elle s'inscrit dans le nouveau modèle d'organisation politique, sécularisé, appuyé sur la puissance publique de l'Etat. Ce dernier ne peut en effet légitimer son pouvoir qu'à travers l'intérêt public qu'il vient servir ${ }^{18}$. Le concept même d'intérêt public sert ainsi à délimiter la sphère étatique de la société civile où règnent les intérêts privés, à une époque où la frontière séparant l'une de l'autre n'est pas nettement tracée ${ }^{19}$. "Intérêt public » et « intérêts privés » forment ainsi une figure "d'extériorité réciproque $»^{20}$ permettant de tracer les limites aux interventions respectives de l'Etat et de la société civile. Aucun ne doit empiéter sur le champ de l'autre.

Garant de l'intérêt public, l'Etat ne peut agir qu'à travers l'administration et les hommes qui la composent. Emerge ainsi la figure du fonctionnaire ${ }^{21}$, autrement appelé «homme citoyen» ou «officier de la Nation ${ }^{22}$ », pour désigner tout homme qui exerce une ou plusieurs fonctions du gouvernement. Dévoué à la chose publique, le fonctionnaire est soumis à un régime juridique spécial de devoirs et de responsabilités réciproques qui implique la subordination complète des intérêts privés à l'intérêt général. Dans le Dictionnaire général et raisonné de Législation, de Doctrine et de Jurisprudence (...), Armand Dalloz affirme

\footnotetext{
${ }^{14}$ M. HAURIOU, Précis de droit administratif et de droit public, 1903, p. 552.

${ }^{15}$ M. HaURIOU, op. cit., p. 554.

${ }^{16} \mathrm{M}$. Hauriou, op cit. p. 556 qui rejette clairement la thèse d'une situation contractuelle du fonctionnaire vis-à-vis de l'administration,

${ }^{17} \mathrm{G}$. CONAC, préf. in C. KAFTANI, La formation du concept de fonction publique en France, LGDJ, 1998, p. XXII.

${ }^{18}$ P. MOOR, Pour une théorie micropolitique du droit, PUF, 2005, p. 83.

${ }^{19}$ P. MOOR, op. cit., p. 88.

${ }^{20}$ P. MOOR, op. cit., p. 103.

${ }^{21}$ Faisant écho à l'abolition des privilèges et à l'ouverture de la fonction publique à tous les citoyens (Décret 4 août 1789, art. 6 Déclaration des droits de l'homme et du citoyen).

${ }^{22}$ C. KAFTANI, op. cit., p. 66.
} 
ainsi que «c'est de la société et de l'Etat que les fonctionnaires tiennent leur caractère et leurs pouvoirs; en échange et à raison même de cette autorité les fonctionnaires sont soumis à des plus fortes garanties et à une plus sévère responsabilité »; les garanties et responsabilités mises en place visent plus à défendre la fonction que la personne du fonctionnaire ${ }^{23}$ et concrétisent ainsi l'idée selon laquelle la fonction publique préexiste à la personne de fonctionnaire ${ }^{24}$. C'est ici l'origine de la création, à l'article 175 du code pénal de 1810, du délit d'ingérence, rangé dans le Titre I du Livre III consacré au « crimes et délits contre la chose publique» tenus comme "une classe spéciale d'infractions " en raison de la "qualité de l'agent et des devoirs particuliers qu'il enfreint ».

\section{B. La création et l'interprétation large du délit d'ingérence}

L'article $175^{25}$ doit protéger l'intérêt public contre «Tout fonctionnaire, tout officier public, tout agent du Gouvernement, qui, soit ouvertement, soit par actes simulés, soit par interposition de personnes, aura pris ou reçu par quelque intérêt que ce soit, dans les actes, adjudications, entreprises ou régies, dont il a ou avait, au temps de l'acte, en tout ou en partie, l'administration au la surveillance (...) ». La sanction est " un emprisonnement de six mois au moins, et de deux ans au plus » ainsi qu'une " amende qui ne pourra excéder le quart des restitutions et des indemnités, ni être au-dessous du douzième. Il sera de plus déclaré à jamais incapable d'exercer aucune fonction publique. La présente disposition est applicable à tout fonctionnaire ou agent du Gouvernement qui auras pris un intérêt quelconque dans une affaire dont il était chargé d'ordonnancer le paiement ou de faire la liquidation $»$.

Ainsi rédigé, l'article 175 de l'ancien code pénal sanctionne la violation, par le fonctionnaire, de son devoir de probité, c'est-à-dire de loyauté à l'Etat. Le délit, véritable "trahison de la confiance de l'Etat ", constitue un "abus de pouvoir " ou encore un "abus de la confiance publique $»^{26}$ entendu comme un abus de l'autorité qui lui est confiée ; parce que ses sources sont exclusivement les règles fondatrices de la sphère publique, dans une société où celle-ci est appelée à coexister avec la sphère privée dans une certaine ignorance réciproque ${ }^{27}$, le délit s'analyse comme le versant répressif de l'obligation de non-immixtion. Par suite, l'entier délit se consume dans la constatation d'une immixtion ou d'un risque d'immixtion, par l'agent public, dans la sphère privée, pour la partie - c'est une condition - placée sous son autorité. C'est reconnaître que le délit sanctionne une simple situation et non un comportement. Dès lors les conditions sont posées

\footnotetext{
${ }^{23}$ C. KAFTANI, op. cit., p. 108.

${ }^{24}$ C. KAFTANI, op. cit., p. 135.

${ }^{25}$ Code pénal de l'empire français, www.gallica.bnf.fr

${ }^{26}$ F. HÉLIE, Leçons de droit criminel, 1872, Paris, p. 278, n 254.

${ }^{27}$ Livre blanc, 2008, p. 56.
} 
pour une interprétation objective et large du délit ${ }^{28}$. De fait, tel que fixée par la jurisprudence, l'ingérence est dès l'origine un délit préventif (délit-obstacle) dont la constitution n'exige pas de préjudice de l'administration, ni d'enrichissement de son auteur ${ }^{29}$; l'immixtion est caractérisée par tout intérêt, de nature quelconque $^{30}$, pris ou reçu, directement ou indirectement, par une action ou une simple abstention ${ }^{31}$.

La délimitation du champ du délit par les juges participe directement à la définition de la fonction publique, encore en construction. C'est en effet à travers la mise en œuvre de la responsabilité pénale que sont précisées les notions de "fonctionnaire» et d' "agent $d u$ gouvernement» visées par le délit. Logiquement, le juge pénal va retenir une approche plus fonctionnelle que statutaire et retenir toutes personnes "qui participent à l'exercice de fonctions publiques", qu'elles "soient ou non titulaires d'une parcelle de puissance publique $^{32}$." La situation d'immixtion se cristallise alors sur l'entreprise ou l'affaire sur laquelle l'agent public exerce, dans le cadre de sa mission, une surveillance. Essentielle, cette condition est, dès l'origine, appréciée largement puisque la notion de surveillance est condensée dans celle d'autorité entendue comme la possibilité, pour l'agent public "d'une façon plus ou moins efficace (...) de favoriser l'affaire $\|^{33}$. Dès lors, peu importe que l'agent ait exercé cette autorité seul ou collectivement, qu'il n'ait eu aucun pouvoir de décision; il est par exemple suffisant qu'il ait participé à un projet soumis, ensuite, pour approbation à un organisme ${ }^{34}$. Dans ces conditions, la bonne foi de l'agent, quoique «souverainement constatée» n'écarte pas le délit comme l'affirme la chambre criminelle de la Cour de cassation dans un arrêt du 15 décembre $1905^{35}$ relayé par la doctrine ${ }^{36}$. La solution est, depuis, constante ${ }^{37}$.

\footnotetext{
${ }^{28}$ Simone MAJEROWICZ, « La rémunération des élus, dirigeants de sociétés d'économie mixte : prise illégale d'intérêts ou non-soumission à l'article 432-12 du Code pénal ? ", JCP. G., Etude n 50, 1994, doctr. 3808.

${ }^{29}$ Crim. 18 juin 1996, $\mathrm{n}^{\circ} 95-82759,-27$ nov. 2002, $\mathrm{n}^{\circ} 7066,7$ janv. 1992, $\mathrm{n}^{\circ} 90-87537,-14$ janv. $1992, n^{\circ} 91-83477$.

${ }^{30}$ Matériel, familial ou moral : Cass.crim.5 nov. 1998, $\mathrm{n}^{\circ}$ 97-80419; par déduction : Crim. 20 fév. $1995, \mathrm{n}^{\circ}$ 94-81186.

${ }^{31}$ Crim. $1^{\text {er }}$ mars $1994, n^{\circ} 93-81461,-20$ fevr. $1995, n^{\circ} 94 \_81186 ;-5$ nov. 1998 , Bull. $n^{\circ} 289$.

32 J-M. TOPPIA, Fonction politique et délit d'ingérence, éd. de l'aube, 1996, p. 120. G. ProtièrE, Les conflits d'intérêts en droit public HAL-00823917, \$16; peu importe qu'elle n'entre pas dans la hićrarchic de l'organisation administrative : Crim. 20 nov. 1980, $\mathrm{n}^{\circ} 79-92715,-21$ nov. $1985, \mathrm{n}^{\circ} 84-$ 93133, -10 févr. $1988, n^{\circ} 86-93258,-5$ nov. $1998, n^{\circ} 97-80419$.

${ }^{33}$ J-M. Toppia, op. cit., p. 190.

${ }^{34}$ Crim. 14 janv. 1943, Bull.crim.1943, n 4 . Eg. Crim. 7 oct. 1976, RSC, 1977.325, obs. Vitu.

${ }^{35}$ D.1907.1, p. 195 et s. Selon le conseiller-rapporteur la «loi punit le seul fait matériel de l'ingérence du fonctionnaire dans une affaire incompatible avec sa qualité, in Com. s/Crim. 15 déc. 1905 , préc., spéc., p. 199. V. ég., Crim., 15 avr. 1848, Bull. crim. n 120 , 10 avr. 1897, cité par Mercier, Rapp. préc., p. 199.

${ }^{36}$ E. DALlOZ et Ch. H. VERGÉ, Code pénal annoté, jurisprudence générale, Paris, 1881, art. 175 CP, $\S 2$ et $\mathrm{s}$.

${ }^{37}$ Crim. 15 avr. 1848, Bull. crim., 1848, n 120, -11 mars 1976, Bull. crim, 1976, JCP. 1976. II 18460. Rennes, 21 mai 1979, note D. Truchet, Rev. Jud. Ouest, 198-1 ; ég. Crim. 17 févr. 1987, $n^{\circ} 85-96223$.
} 
En 1990, des auteurs ${ }^{38}$ écrivaient encore que le dol général "est présumé de manière irréfragable en raison des obligations même du fonctionnaire » et de conclure que "la bonne foi n'est pas exclusive du délit »"

L'adoption du nouveau code pénal de 1992 est néanmoins l'occasion, pour le législateur, d'opérer une redéfinition, en réalité partielle, de l'infraction dans un nouvel article 432-12.

\section{La fausse rupture du code pénal de 1992}

Le nouvel article 432-12 du code pénal punit de cinq ans d'emprisonnement et d'une amende de $500000 €^{40}$ le fait « par une personne dépositaire de l'autorité publique ou chargée d'une mission de service public ou par une personne investie d'un mandat électif public, de prendre, recevoir ou conserver, directement ou indirectement, un intérêt quelconque dans une entreprise ou dans une opération dont elle a, au moment de l'acte, en tout ou partie, la charge d'assurer la surveillance, l'administration, la liquidation ou le paiement ». Rangé, après les atteintes aux personnes et aux biens, dans le Livre IV du code pénal $^{41}$ consacré aux «Crimes et délits contre la nation, l'Etat et la paix publique », le nouvel article ne vise plus une atteinte « à la chose publique » par une " ingérence » de l'agent public dans le champ privé, mais un " manquement au devoir de probité (section III) par "une prise illégale d'intérêts » portant « atteinte à l'administration publique » (Chapitre II). Cependant, tant pour la doctrine $^{42}$ que la jurisprudence ${ }^{43}$, le nouvel article s'inscrit dans la continuité du délit d'ingérence de l'ancien article $175^{44}$. Un auteur ${ }^{45}$, en écho à la doctrine dominante, l'affirme : "Au-delà du changement de vocabulaire, il n'y a pas de changement de fond : l'infraction reste la même dans toutes ses composantes de droit et de fait. " En visant désormais les " dépositaires de l'autorité publique, personnes investies d'un mandat électif public ou chargée d'une mission de service public ", il ne fait que consacrer la jurisprudence antérieure en confirmant l'approche plus fonctionnelle que statutaire de l'agent public. Seule compte la participation à la gestion des affaires publiques ${ }^{46}$ indépendamment de

\footnotetext{
${ }^{38}$ M. Delmas-Marty et G. Guidicelli-Delage, Droit pénal des affaires, $4^{\text {ème }}$ éd., 1990, p. 298.

${ }^{39}$ Crim. 25 juin 1996, n 95-80592 : l’élément moral du délit résulte du manquement des prévenus à l'obligation, que leur imposait leur mandat électif, de vérifier la régularité des engagements qu'ils prenaient pour cux-mêmes ou pour la communc. Eg. Crim. 18 oct. 1994, n 93-83396.

${ }^{40}$ Dont le montant peut être portć au double du produit tiré de l'infraction.

${ }^{41}$ Titre III relatif aux atteintes à l'autorité de l'Etat.

${ }^{42}$ W. JEANDIDIER, art. préc.. J-M. TOPPIA, op. cit., , p. 43.

${ }^{43}$ Pour un délit constitué par le scul abus de fonction indépendamment d'intérêt personnel: cass.crim. 23 juill. $2014, \mathrm{n}^{\circ} 13-82193$.

${ }^{44}$ F. DESPORTES et F. LE GUNEHEC, «Présentation des dispositions du nouveau code pénal », JCP G., $n^{\circ} 41,7$, oct. 1992, doctr. 3615 : la notion d'opération qui se substituc à l'ćnumération " actes, adjudications, régies » est en effet considéréc comme étant plus large puisqu'clle vise l'ensemble des opérations du secteur privé et du secteur public; J-M. TOPPIA, op. cit., p. 211. De même, les dérogations prévues en faveur des petites communes sont largement étendues.

45 F. KAPPEHLOFF LANCON, "Les délits d'ingérence et de prise illégale d'intérêts ", $L P A$, $15 / 02 / 1995, n^{\circ} 20$, p. 13 et $s$.

${ }^{46} \mathrm{~J}$-M. TOPPIA, op. cit., p. 120.
} 
la place, hiérarchique ou non, de la personne dans l'administration ${ }^{47}$. Est même noté un élargissement du délit puisque le texte ajoute aux notions de " prise » et de "réception » celle de « conservation », par le prévenu, d'un intérêt. En écho, la jurisprudence va continuer de retenir une interprétation large du délit. Ainsi et bien que le code pénal ait pris soin de poser, dans un nouvel article 121-3, qu' « il n'y a point (...) de délit sans intention de le commettre ", le délit de prise illégale d'intérêts reste un délit objectif, la constatation de l'élément intentionnel étant purement formelle. Concrètement, l'intention coupable est caractérisée par « le seul fait que l'auteur a accompli sciemment l'acte constituant l'élément matériel ${ }^{48}$ quand bien même il aurait pris soin, préalablement à l'acte, de consulter ses avocats pour s'assurer de sa légalité ${ }^{49}$; et s'il était en l'espèce certain que le prévenu ignorait accomplir un acte prohibé par la loi, il aurait dû selon la doctrine, "le savoir, et là réside sa faute, son dol qui est de la sorte présumé ». D'où l'idée que " contrairement à la plupart des infractions pénales, le délit de prise illégale d'intérêts ne pose pas de difficulté dans son élément moral, s'agissant d'une infraction objective dont la réalisation ne nécessite aucune intention frauduleuse ${ }^{50}$. Surtout, poussant le raisonnement plus avant, la chambre criminelle ${ }^{51}$ de la Cour de cassation va déduire du même acte, la preuve de la prise d'un intérêt et celle de la surveillance de l'affaire ou entreprise dans laquelle l'intérêt a été pris. Concrètement, la délibération de l'agent public, jugée fautive, caractérise à la fois la surveillance de l'opération contestée et la prise d'intérêt dans cette opération.

Finalement, l'interprétation jurisprudentielle du délit de prise illégale d'intérêts reste, deux siècles après sa création, profondément marqué par son rattachement à une administration publique au service de l'intérêt général qui impose de tenir le fonctionnaire à l'écart "des antagonismes socio-économiques s'inscrivant dans les rapports de production qui risquent de compromettre l'unité interne de l'appareil étatique ${ }^{52}$.

Pourtant, dès les années 1980 , un auteur ${ }^{53}$ invite à une modification du texte du délit d'ingérence aux motifs que «l'administration actuelle connait une

\footnotetext{
${ }^{47} \mathrm{~V}$. pour une personne morale de droit privé " exerçant une mission de service publique », Crim. 24 mars $2010, \mathrm{n}^{\circ} 09-81153$.

${ }^{48}$ Crim., 21 nov. 2001, $\mathrm{n}^{\circ}$ 00-87532. Pour un intérêt moral jugé nécessairement distinct de l'intérêt général : Crim. 29 juin 2011, $\mathrm{n}^{\circ} 10-87498$; - 15 juin 2016, $\mathrm{n}^{\circ} 15-81124$. Contra: Poitiers, 16 mai 2013, n 13/00170, note B. Fleury, Prise illégale d'intérêt : le grand retour de l'intention ? préc., qui, pour le cas d'espèce, relève que « analyser » l'intention « constitue une nouveauté qu'il convient de souligner $»$.

${ }^{49}$ Crim. 27 nov. 2002, n 7066 , W. JEANDIDIER, art. préc., qui note que si cette précaution ne suffisait pas à caractériser l'erreur de droit au sens de l'article du code pénal, du moins permettait-elle de prouver la bonne foi du prévenu.

${ }^{50}$ E. DuPIC, « La prise illégale d'intérêts ou le mélange des genres ", note s/Crim. 11 févr. 2009, JCP G.22 juin $2009,44$.

${ }^{51}$ Ainsi, le fait pour le prévenu, conseiller général, d'avoir pris part à un vote au sein de la commission permanente du conseil général caractérise l'acte de prise d'intérêt dans l'affaire incriminée et, simultanément, le pouvoir de surveillance ou d'administration sur celle-ci : Crim., 19 mai $1999, \mathrm{n}^{\circ} 98-80726 ;-24$ mai $2016, \mathrm{n}^{\circ} 14-83895$.

${ }^{52}$ C. KAFTANI, op. cit., p. 156.

${ }^{53}$ D. TRUCHET, note préc., p. 98.
} 
interpénétration profonde des intérêts privés et des intérêts publics » et qu'il "faudrait en tenir compte (...) pour éviter la condamnation de personnes dont l'honnêteté est proclamée par les juges eux-mêmes!". C'est sans doute annoncer que le délit de prise illégale d'intérêts sanctionne désormais la prévention d'un conflit d'intérêts.

\section{LE DÉLIT DE PRISE ILLÉGALE D'INTÉRÊTS OU LA SANCTION DE LA PRÉVENTION D'UN CONFLIT D'INTÉRÊTS}

\section{A. L'ignorance initiale du conflit d'intérêts par le droit positif}

Parce qu'il sanctionne une simple "confusion des genres » ${ }^{54}$, aux fins d'éviter que naisse le soupçon sur la probité de l'agent public, le délit n'exige pas, pour être constitué, un conflit d'intérêts ${ }^{55}$; sans doute celui-ci constitue-t-il une forme patente du délit, pour autant il n'est pas une condition nécessaire à sa constitution. En témoigne l'arrêt de la chambre criminelle de la Cour de cassation du 19 mars 2008 qui ne juge pas nécessaire, pour caractériser le délit, que "l'intérêt pris par le prévenu soit en contradiction avec l'intérêt communal ». Autrement dit, le délit de prise illégale d'intérêt peut résulter autant d'un conflit que d'une confusion d'intérêts ${ }^{56}$.

Cela explique sans doute le constat, fait par la doctrine, que "le conflit d'intérêts $n$ 'existe pas dans notre droit pénal $\aleph^{57}$. Constat qui, jusque récemment encore, irriguait, au-delà du droit pénal, le droit administratif tant la notion de conflit d'intérêt allait «à l'encontre de la conception française de service public et de son horizon indépassable qu'est l'intérêt général $»^{58}$. Ou, pour le dire autrement, "pas de conflit d'intérêts là où gouverne, seul, l'intérêt général »"

Or, ce schéma public est entré dans un temps de crise à partir de la deuxième moitié du XXème siècle. La crise économique liée au choc pétrolier, le retour du libéralisme mais aussi, globalement, la construction de l'Europe et, plus tard, la mondialisation, se conjuguent pour conduire à une réévaluation de la place de l'Etat $^{60}$. Remise en cause, jugée inefficace, l'intervention de l'Etat devient envahissante. Son périmètre et, au-delà, la nécessité et même l'utilité de la chose publique $^{61}$ sont contestés. A l'Etat garant de l'intérêt général se substitue

\footnotetext{
${ }^{54}$ J-M. TOPPIA, op. cit., p. 205.

${ }^{55} \mathrm{G}$. Protière, art. préc.

${ }^{56}$ Eg., Crim. 22 oct. 2008, n 08-82068, -9 févr. 2011, n 10-82988. v. ćg. E. DREYER, comm. s/27 févr. 2013, préc.

${ }^{57}$ D. REBUT, « Les conflits d'intérêts et le droit pénal », Pouvoirs, vol. 147, no. 4, 2013, pp. 123-131, citant M. Hirsch.

${ }^{58}$ E. BOMBERGER, «L'inspiration internationale du droit français en matière de conflits d'intérêt ", Dr. $A d m . \mathrm{n}^{\circ} 7$, juill. 2015, Etude 10.

${ }^{59}$ M. PINAult, "Conflits d'intérêts : glaive ou boomerang ? ", Pouvoirs, vol. 147, no. 4, 2013, pp. 33-44.

${ }^{60}$ J. CheVAllier, L'Etat post-moderne, LGDJ, 2014, p. 26 et s.

${ }^{61}$ C. Vigouroux, Déontologie des fonctions publiques, Dalloz 2013/2014.
} 
progressivement un Etat gestionnaire ${ }^{62}$. L'évolution est portée dès les années 1980 par le mouvement du "New public management ${ }^{63}$ qui propose d'adapter l'administration publique sur le modèle de gestion de l'entreprise privée prônant un net désengagement de l'Etat; la fonction publique ${ }^{64}$ est invitée à se rapprocher de l'entreprise en répondant à l'impératif d'efficacité ${ }^{65}$ et de transparence qui la concerne "au même titre que le secteur privé » ${ }^{66}$. Et depuis les années 2000, se développe à côté de la fonction publique traditionnelle, de carrière et statufiée, une fonction publique parallèle reposant sur une logique contractuelle $^{67}$ et qui emprunte de plus en plus au droit européen ${ }^{68}$. Dès lors, la distinction du secteur public et du secteur privé, sur lequel l'Etat a construit sa légitimité, se fissure pour laisser place à une conception plus souple marquée par la porosité des deux domaines. La conduite des affaires publiques est en effet de plus en plus opérée au travers de relations complexes entre acteurs privés et acteurs publics ${ }^{69}$. L'évolution traduit en outre une certaine "décomposition ${ }^{70}$ de l'intérêt public éclaté en de multiples intérêts publics divergents ${ }^{71}$ comme l'intérêt public local ou l'intérêt collectif territorial ${ }^{72}$, et au-delà encore, l'intérêt public européen, laissant apparaître un conflit entre intérêts publics. Finalement d'une opposition binaire entre intérêt général et intérêts privés, on est passé à un schéma d'emboitement des différents intérêts généraux et privés, éclatés dans le temps et dans l'espace et adossés à des institutions légitimes ${ }^{73}$ au point que soit proposée "une définition globalisante du conflit d'intérêts, commune à la sphère publique et à la sphère privée ${ }^{74}$. Ce mouvement trouve un écho législatif. Dès les années 1960, le législateur ouvre une brèche ${ }^{75}$ dans la sanction de la prise illégale d'intérêt en autorisant certaines opérations dans les petites communes, dérogations qui seront élargies par le code pénal de 1992 et ce, afin de permettre un «juste équilibre entre la nécessaire prise en compte des préoccupations des élus et l'impérieuse nécessité (...) d'assurer la moralisation de la vie publique $»^{76}$. Il se confirme, dans les années $1980-1990^{77}$ avec, notamment, la loi

\footnotetext{
${ }^{62}$ P. MOOR, op. cit., p. 93.

${ }^{63}$ Mouvement qui a supplanté le mouvement de «nouvelle gestion publique » porté, à travers l'école française de « management public ».

${ }^{64}$ C. Vigouroux, Déontologie des fonctions publiques, Dalloz, 2013/2014, p.24, $\$ 01.101$.

${ }^{65}$ J. Chevallier, op. cit., p. 70.

${ }^{66} \mathrm{Ce}$ mouvement trouve un aboutissement dans la loi organique $\mathrm{n}^{\circ} 2001-692 \mathrm{du}^{\mathrm{er}}$ août 2001 relative aux lois de finances.

${ }^{67}$ B. BARRAUD, Du statut au contrat : vers une fonction publique "déstatufiée », HAL-01367449, 2016. V. supra le code pénal de 1992 qui place la protection des intérêts de l'Etat, de la Nation après celle des personnes et des biens.

${ }^{68}$ S. VOKO, Les atteintes à la probité, thèse Paris 1, 2016, p. 15, HAL-01522452.

${ }^{69}$ J.B. AUBY, «Conflits d'intérêts et droit administratif », Dr. Adm. № 12., déc. 2010, étude 24.

${ }^{70}$ P. MOOR, op. cit., p. 99.

${ }^{71}$ P. MOOR, op. cit., p. 100.

${ }^{72} \mathrm{~K}$. SIBIRIL, Définition de la notion d'intérêt en droit administratif française, Thèse Université de Bretagne, 2012, p. 12, HAL-01162440.

${ }^{73}$ K. SIBIRIL, p. 12.

${ }^{74}$ M. MUSTAPHA. « La lutte contre les conflits d'intérêts : essor de la transparence ou règne de la méfiance? ", Pouvoirs, vol. 147, no. 4, 2013, pp. 17-32.

${ }^{75}$ Loi n ${ }^{\circ}$ 67-467 du 17 juin 1967 ; l'autorisation de la loi est élargie par le code pénal de 1992.

${ }^{76} \mathrm{~J}-\mathrm{M}$. TOPPIA, op. cit., p. 245.
} 
$\mathrm{n}^{\circ} 83-634$ du 13 juillet $1983^{78}$ qui autorise l'agent public à exercer une activité lucrative dès lors qu'elle est compatible avec ses fonctions ${ }^{79}$. C'est dans ce contexte, révélant une transformation des démocraties représentatives, que la problématique des conflits d'intérêts fait irruption.

\section{B. La prise de conscience croissante des conflits d'intérêts}

Dès le début des années 2000, le Conseil de l'Europe puis l'OCDE ${ }^{80}$ proposent une définition du conflit d'intérêts aux fins de définir un mode de régulation des nouveaux types de relations et formes de collaboration entre le secteur public et le secteur privé. S'inspirant de ce mouvement ${ }^{81}$, deux commissions sont chargées en France, en 2010 (Commission Sauvé ${ }^{82}$ ) et 2012 (Commission Jospin ${ }^{83}$ ) de faire des propositions relatives à la prévention des conflits d'intérêts dans la vie publique. Leurs propositions aboutiront à la loi n 2013-907 du 11 octobre 2013 relative à la transparence de la vie publique ${ }^{84}$ qui, pour la première fois, donne une définition du conflit d'intérêts ${ }^{85}$ récemment reprise dans la loi $n^{\circ} 2016-483$ du 20 avril 2016 relative à la déontologie et aux droits et obligations des fonctionnaires ${ }^{86}$. De son côté, le Conseil d'Etat semble, selon un auteur, "acter le changement politique et normatif ${ }^{87} \gg$. En effet, la situation de prise illégale d'intérêts par un agent public constitue un cas classique d'ouverture du recours en annulation pour excès de pouvoir. Le juge administratif est alors amené à contrôler la matérialité des faits reprochés à l'agent dans le cadre d'une procédure disciplinaire. Or, à partir de 2005, l'expression "conflit d'intérêts » supplante, dans la jurisprudence, celle de «prise illégale d'intérêts » qui décroit proportionnellement à sa progression. C'est dire que dans le champ administratif, la probité prend désormais la figure d'un conflit d'intérêts.

\footnotetext{
${ }^{77}$ Loi n ${ }^{\circ} 88-227$ du 11 mars 1988 relative à la transparence financière de la vic politique, Loi $n^{\circ}$ 91-3 du 3 janv. 1991 relative à la transparence et à la régularité des procédures de marchés et soumettant la passation de certains contrats à des règles de publicité et de mise en concurrence, Loi $n^{\circ} 93-122$ du 29 janv. 1993 relative à la prévention de la corruption et à la transparence de la vic économique et des procédures publiques (dite loi Sapin I.) V. J. BÉNOIT, Rép. Dalloz, Collectivités territoriales, Chap. 4, Règles de transparence et de probité, 2014.

${ }_{78}^{78}$ Portant droits et obligations des fonctionnaires.

${ }^{79} 25 \S 3^{\circ}$.

${ }^{80}$ Recommandation du Conscil sur les lignes directrices pour la gestion des conflits d'intérêts dans le scrvicc public, 28 mai 2003.

${ }^{81}$ V. ég., loi $\mathrm{n}^{\circ}$ 2007-148 du 2 fév. 2007 de modernisation de la fonction publique (Chapitre III, règles de déontologie) qui créc une commission de déontologic.

${ }^{82}$ Décr ; n² 2010-1072 du 10 sept. 2010 instituant une commission de réflexion pour la prévention des conflits d'intérêts dans la vic publique.

83 Décr. $\mathrm{n}^{\circ}$ 2012-875 du 16 juill. 2012 portant création d'une commission de rénovation et de déontologic de la vic publique.

${ }^{84}$ V. ég., Loi n ${ }^{\circ}$ 2007-148 du 2 févr. 2007 de modernisation de la fonction publique.

${ }^{85}$ Art. $2:$ « (...) constitue un conflit d'intérêts toute situation d'interférence entre un intérêt public et des intérêts publics ou privés qui est de nature à influencer ou à paraître influencer l'exercice indépendant, impartial et objectif d'une fonction ".

${ }^{86}$ Art. 2 modifiant l'art 25 bis. I de la loi n 83-634 préc.

${ }^{87}$ G. PROTIĖRE, art. préc.
} 
Par un effet miroir, les recours et propositions se multiplient pour redéfinir l'intérêt visé dans le délit, de manière à mieux marquer son opposition à l'intérêt public. Déjà, les Commissions Sauvé et Jospin (préc.) concluent à la même nécessité de resserrer le texte du délit de prise illégale d'intérêts, jugé trop large, et de substituer, à la notion d'« intérêt quelconque » pris par l'agent public, celle, plus étroite, d'" intérêt de nature à compromettre l'impartialité, l'indépendance ou l'objectivité de l'agent public ${ }^{88}$. Simultanément, plusieurs propositions de $1 \mathrm{l}^{89}{ }^{89}$ visant également à «clarifier le champ du délit de prise illégale d'intérêts » sont adoptées ; elles tendent, à l'instar des propositions des commissions Sauvé et Jospin, à remplacer, dans le texte du délit, la notion "d'intérêt quelconque » par une définition plus étroite de l'intérêt pris en le ramenant à " un intérêt personnel distinct de l'intérêt général " ou à un intérêt " de nature à compromettre l'impartialité, l'indépendance ou l'objectivité de la personne». Dans tous les cas, ainsi modifié, le délit marque une opposition nette entre l'intérêt pris par l'agent public et l'intérêt qu'il doit protéger; que le conflit d'intérêts soit réel ou potentiel, c'est dans tous les cas ramener le champ du délit de prise illégale d'une confusion à un conflit d'intérêts. Toutes ces propositions sont cependant restées vaines. Il n'en demeure pas moins dorénavant nécessaire de ramener le délit de prise illégale d'intérêts dans le champ du conflit d'intérêts.

\section{La nécessité pour le délit de prise illégale d'intérêts de se saisir clairement du conflit d'intérêts}

Concomitamment à ce mouvement législatif, deux questions prioritaires de constitutionnalité (QPC) ont été posées et transmises à la Cour de cassation, en 2011 puis 2014. La première interrogeait sur la conformité des dispositions de l'article 432-12 CP aux principes constitutionnels de la légalité pénale (art. 34 de la Constitution et art. 8 de la Déclaration des droits de l'homme et du citoyen) dès lors qu'elles ne définissent pas en termes suffisamment clairs et précis le délit. La Cour de cassation a rendu, le 30 novembre $2011^{90}$ un arrêt de non-lieu à renvoi jugeant la rédaction du texte conforme aux principes de précision et de prévisibilité de la loi pénale dont elle permet de déterminer le champ d'application. La deuxième, plus précise, contestait la conformité des dispositions de l'article 432-12 CP, au principe constitutionnel de nécessité et proportionnalité de la peine (art. 8 DDHC préc.) ainsi qu'au principe de légitimité de l'incrimination (art. 5 DDHC), en ce que, telles qu'interprétées de

\footnotetext{
${ }^{88}$ Rapport J-M. SAUVÉ, Pour une nouvelle déontologie de la vie publique, 2011, www. Conflitsinterets.fr, p. 116 qui seul vise en plus «l'objectivité » de la personne ; Rapport L. JOSPIN, Pour un renouveau démocratique, 2012, ladocumentationfranaise.fr, p. 91.

${ }^{89}$ Proposition Saugey visant "à clarifier le champ des poursuites de la prise illégale d'intérêts ", Sénat 24 juin 2010; v. Rapport $\mathrm{n}^{\circ} 519$, texte adopté $\mathrm{n}^{\circ} 133,2009-2010$; La même formulation a été reprise un an plus tard dans le cadre d'une proposition de loi "visant à renforcer l'attractivité et à faciliter l'exercice du mandat local », adoptée 30 juin 2011 (Sénat, texte adopté $n^{\circ} 154,2010-2011$ ) puis par l'adoption en séance d'un amendement de F. Pillet dans le cadre de l'examen d'une nouvelle proposition de loi «visant à faciliter l'exercice, par les élus locaux, de leur mandat », adoptée par le Sénat le 29 janv. 2013 (texte $\mathrm{n}^{\circ} 78,2012-2013$ ).

${ }^{90} \mathrm{~N}^{\circ} 11-90093$.
} 
façon constante par la Cour de cassation, elles incriminent des comportements "qui ne portent atteinte ni à l'intérêt général, ni à des intérêts particuliers, mais constituent seulement une potentialité de faute». Si là encore, la Cour de cassation a rendu, le 19 mars $2014^{91}$ un arrêt de non-lieu à renvoi, sa motivation est plus marquante puisque, pour dire la QPC dénuée de " caractère sérieux " et conforme aux principes constitutionnels évoqués, elle affirme que le législateur incrimine ici le fait, "par une personne exerçant une fonction publique, de se placer dans une situation où son intérêt entre en conflit avec l'intérêt public dont elle a la charge", qu'il a entendu ainsi "garantir, dans l'intérêt général, l'exercice indépendant, impartial et objectif des fonctions publiques ». La décision de la Cour de cassation, qui se rapproche ainsi des diverses propositions doctrinales ou législatives, semble ici valider l'exigence d'un conflit d'intérêts ou d'un simple risque, par la situation prise, de conflit d'intérêts de nature à créer un soupçon dans la probité de l'agent public. Toutefois, elle est clairement revenue sur sa position, dans sa jurisprudence de 2016 et $2017^{92}$ en jugeant que la prise illégale d'intérêts couvre non seulement le conflit d'intérêts mais aussi la convergence d'intérêts. La jurisprudence de la Cour de cassation demeure ainsi marquée d'incertitude et d'imprévisibilité, voire d'une certaine contradiction. A cela s'ajoute une distorsion entre le droit pénal et le droit administratif. La mutation de l'Etat et, partant, la reconfiguration ${ }^{93}$ de la fonction publique se traduit par une nouvelle appréhension de l'éthique de l'action publique. Une nouvelle déontologie de l'agent public émerge dont les obligations, pour l'agent public, sont moins la contrepartie de ses prérogatives de puissance publique que ses obligations de répondre aux exigences d'une charte qualité du service offert au public. Concrètement, la primauté de l'intérêt général soutenant le rapport entre l'agent public et l'Etat doit être articulée avec les exigences nouvelles de transparence et d'efficacité d'une administration publique sur laquelle les citoyens exercent un contrôle ${ }^{94}$. Ces exigences nouvelles traduisent une forme de «dé-distanciation ${ }^{95}$ » de l'administration du reste de la société dans un contexte de porosité entre le secteur public et le secteur privé ${ }^{96}$. Sans doute ce changement a-t-il été perçu par les juges de la Cour d'appel de Bordeaux lorsqu'ils affirment, en 2009, que les délits de prise illégale d'intérêts, complicité et recel de ce délit, sanctionnent des "manquements aux règles de transparence, de prudence et de sincérité qui doivent être appliquées lorsqu'on gère des fonds publics ${ }^{97}$.

Mais pour n'avoir pas, du moins pas clairement, saisi ce mouvement, la jurisprudence de la chambre criminelle entre en dissonance avec celle de la jurisprudence administrative. Ainsi, en droit administratif, la délibération locale est entachée d'illégalité lorsqu'un "conseiller intéressé » à une affaire y a pris

\footnotetext{
${ }^{91} \mathrm{~N}^{\circ} 14-90001$.

${ }^{92}$ Crim. 28 sept. 2016, ${ }^{\circ} 15-83467$. Eg. Crim. 9 nov. 2016, $n^{\circ} 15-86183,-15$ juin $2016, n^{\circ} 15-$

81124, - 22 fćv. 2017, n 16-82039, -9 avr. 2005, n 14-81912.

${ }^{93}$ V. J. Chevallier, op. cit., p. 21 et s.

${ }^{94}$ B. BARRAUD, art. préc., p. 7.

95 J. CHEVALLIER, « Le mythe de la transparence administrative ", www.u-picardic.fr, p. 255.

${ }^{96}$ G. PROTIĖRE, art. préc., \$21.

${ }^{97}$ Bordcaux, 13 janv. 2009, 08/00398.
} 
part $^{98}$. Or la notion de "conseiller intéressé», sur laquelle le Conseil d'Etat exerce un contrôle de qualification, suppose, à l'inverse du droit pénal, d'une part un intérêt qui ne se confond pas avec les intérêts de la généralité des habitants de la commune ; concrètement, le Conseil d'Etat a précisé qu' «il n'y pas d'intérêts à l'affaire lorsqu'il s'agit d'un intérêt attaché à la qualité d'habitant ou de contribuable de la commune, sans être distinct de l'intérêt général de la commune ${ }^{99}$; la notion suppose d'autre part une influence effective de la participation du conseiller intéressé ${ }^{100}$. La dissonance paraît plus forte encore lorsque l'élu siège dans une association ou dans une société d'économie mixte locale (SEML) en sa qualité de représentant de la collectivité. S'agissant d'une association, la Cour de cassation a jugé ${ }^{101}$, contrairement à la jurisprudence administrative, que l'intérêt pris par des élus municipaux, en participant aux votes de subventions bénéficiant aux associations qu'ils président, entre dans le champ du délit de prise illégale d'intérêts quand bien même l'association poursuivrait un but d'intérêt général et l'élu présiderait l'association ès qualités. S'agissant des SEML, la loi impose aux collectivités territoriales actionnaires de détenir plus de la moitié des voix dans les organes délibérants (art. L 1522-1 CGCT), celles-ci devant alors désigner les élus locaux qui les représenteront ${ }^{102}$. Aussi, le législateur de $2012^{103}$, précisant le rôle des élus locaux représentants au sein de la SEML, est venue circonscrire les cas d'intéressement en décidant que les élus ne sont pas considérés "comme intéressés à l'affaire (...) lorsque la collectivité (...) délibère sur ses relations avec la SEML ». Or, le conseil d'Etat interprète largement la notion de «relations avec la SEML » et juge que les élus qui délibèrent sur un projet de convention portant attribution à une SEML d'un contrat public ne sont pas « intéressés ». Toutefois, l'interprétation stricte du Conseil d'Etat de la notion d' "élu intéressé » ne protège pas d'une éventuelle responsabilité pénale pour prise illégale d'intérêts ${ }^{104}$; sans doute peut-on opposer ici le principe d'autonomie du droit pénal, lequel sanctionne ici un délit et non un acte illégal. Mais dans la mesure où l'histoire et l'interprétation du délit sont étroitement associées à la construction et l'évolution de la fonction publique, notre propos est d'inviter à une réflexion qui englobe le droit pénal et le droit administratif, cela afin que la sanction pénale soit mieux et effectivement appliquée.

\footnotetext{
${ }^{98}$ Art. L2131-11 Code général des collectivités territoriales (CGCT).

${ }^{99} \mathrm{CE}, 20$ janv. 1989, n ${ }^{\circ} 75442$. Sur la question, B. Fleury, « Nul ne peut servir deux maîtres à la fois "), art. préc.

$100 \mathrm{~J}$-Ch. VideLin et Ph. YolKA, note s/CE 17 nov. 2010, n 338338, JCP A $2011, \mathrm{n}^{\circ} 1$. J-M. BRIGANT, «Affaires, conflits d'intérêts, probité,... cachez cette prise illégale d'intérêts que je ne saurais voir ", Droit pénal $\mathrm{n}^{\circ} 1$, Janv. 2012, étude 3. Du même auteur, Contribution à l'étude de la probité, Université Paris I, 2009.

${ }^{101}$ Crim. 22 oct. 2008 , préc. supra.

102 Art. L 225-20 CCom. et art. L 1524-5 CGCT.

${ }^{103}$ L. $\mathrm{n}^{\circ} 2002-\mathrm{I}$ du 2 janv. 2002 relative à la modernisation du statut des SEML.

${ }^{104}$ S. BRAMERT, « Le statut des élus locaux administrateurs de SEML (...) », JCP A 2013, note s/CE 10 déc. $2012, n^{\circ} 354044$.
} 
A la lumière de ces différentes évolutions, il semblerait opportun de ramener le délit de prise illégale d'intérêts dans le champ du conflit d'intérêts. Il permettrait alors de sanctionner la situation de conflit d'intérêt d'intérêts, réel ou potentiel, d'un agent public dès lors que cette situation menace la partialité de la décision publique. Mieux défini, le délit pourrait être effectivement appliqué et les citoyens plus sensibles aux condamnations pénales, notamment celles touchant les élus.

Mais, et le propos sera conclusif, l'on peut craindre que les développements proposés pour une rénovation du délit de prise illégale d'intérêts ne soient déjà dépassés. Il faut d'abord espérer qu'ils seront lus dans leur juste dimension, c'est-à-dire autrement que dans un raisonnement binaire qui conduirait à réduire l'analyse en un positionnement - ridicule - en faveur ou contre la sanction pénale, celle-ci étant évidemment mais strictement nécessaire. Ensuite, la réflexion sur la probité de la vie publique semble portée par un mouvement de gouvernance globale, transnationale et moralisateur. Le projet de loi «de moralisation de la vie publique», rebaptisé projet de loi "rétablissant la confiance dans l'action publique" et actuellement porté par le ministre de la justice participe de ce mouvement. S'ajoutant aux nombreuses lois déjà votées en ce domaine, il vise cette fois à créer «le choc de confiance » dont a besoin notre vie publique ${ }^{105}$ et passe - nécessairement- par un renforcement de "l'exigence de probité des élus du point de vue des condamnations pénales ${ }^{106}$ " mais aussi par la création d'une "Banque de la démocratie» pour assurer une saine gestion du financement des partis politiques. Et tel le réverbère éclairant le pavé, le "baromètre de la confiance en politique » alimente l'étude d'impact de la $\mathrm{loi}^{107}$ dont la rigueur scientifique ne va pas au-delà de ce que peuvent donner les chiffres. La perplexité du juriste, devant un tel arsenal construit en seulement quelques jours, s'effacera sans doute devant l'émergence d'une culture transnationale de "l'intégrité publique à l'échelon de l'ensemble de la société en partenariat avec le secteur privé", elle-même s'appuyant sur une "une culture institutionnelle de la transparence », et s'intégrant plus largement dans " culture mondiale d'intolérance à la corruption ${ }^{108}$.

\footnotetext{
${ }^{105} \mathrm{~V}$. Exposé des motifs.

${ }^{106}$ Exposé des motifs, art. $1^{\mathrm{cr}}$.

${ }^{107}$ Etude d'impact 14 juin 2017.

${ }^{108}$ Ibidem.
} 